\title{
Use of Contact Force Mapping to identify low Voltage Bridging to guide radiofrequency ablation of slow pathway: A single Center Study
}

Nachiket Apte $^{1}$, Ruchi Bhandari ${ }^{2}$, Catherine Vanchiere ${ }^{3}$, Parinita Dherange ${ }^{4}$, Jason Mackowiak $^{5}$, Narendra Duddyala ${ }^{4}$, Ryan Jones ${ }^{4}$, Ian Law ${ }^{6}$, Daniel Morin ${ }^{7}$, and Paari Dominic $^{4}$

${ }^{1}$ University of South Dakota Sanford School of Medicine

${ }^{2}$ West Virginia University - Health Sciences Campus

${ }^{3}$ Temple University School of Medicine

${ }^{4}$ LSUHSC Shreveport

${ }^{5}$ LSU Shreveport

${ }^{6}$ University of Iowa

${ }^{7}$ Ochsner Medical Center

September 21, 2020

\begin{abstract}
Introduction: Ablation of slow pathway (SP) for AV nodal reentry tachycardia (AVNRT), by visualization of low voltage bridges using non-contact force sensing (CFS) catheters and cryo-ablation has been described. This study aims to demonstrate the use of voltage mapping using CFS catheters in patients with AVNRT undergoing SP modification by Radiofrequency ablation (RFA). Methods Three-dimensional mapping was performed using CFS catheter in adult patients undergoing AVNRT ablation. Voltage values from intracardiac recordings were adjusted until a low voltage bridge was observed. Both electro-anatomical data and low voltage bridges were used to guide ablation. Distance from the earliest His signal to the site of SP (His-SP) and the area of the Koch's triangle (KTArea) was measured. Results A total of 41 adult patients ( $63 \%$ female, $48 \pm 15$ years) underwent RFA using CFS catheters. Median follow up was 12 months. There were no recurrences in any patients. When indexed to body surface area (BSA), both mean His-SP distance $(\mathrm{p}=0.038)$ and mean KTArea $(\mathrm{p}=0.019)$ was greater in patients with atypical AVNRT $(n=5)$ than in patients with typical AVNRT $(n=32)$. Conclusion RFA of AVNRT by visualization of low voltage bridges using CFS is effective. In patients with atypical AVNRT, SPs are particularly distant from the His region. Patients with atypical AVNRT have a larger Koch's triangle than patients with typical AVNRT.
\end{abstract}

\section{Hosted file}

Voltage Bridging in SP modification 9-20-2020.docx available at https://authorea.com/users/ 360734/articles/482284-use-of-contact-force-mapping-to-identify-low-voltage-bridging-toguide-radiofrequency-ablation-of-slow-pathway-a-single-center-study 